\title{
Mobile Phones as Tool to Increase Communication and Location Awareness of Users
}

\author{
Albrecht Schmidt, Paul Holleis \\ Embedded Interaction Group \\ Media Informatics, LMU Munich \\ Germany \\ http://www.hcilab.org/ \\ \{albrecht, paul\}@hcilab.org
}

\author{
Jonna Häkkilä \\ Nokia Multimedia \\ Yrttipellontie 6 \\ 90230 Oulu, Finland \\ jonna.hakkila@nokia.com
}

\author{
Enrico Rukzio, Richard Atterer \\ Media Informatics, \\ LMU Munich, Germany \\ \{Enrico.Rukzio, \\ Richard.Atterer\}@ifi.Imu.de
}

\begin{abstract}
Mobile phones have become a ubiquitous technology and for many people a daily companion. The prime function is to enable communication and information access anywhere and anytime, but the fact that the phone is accompanying the user makes it an interesting platform for building persuasive applications and for use as ambient display. It offers means for collecting information and sensing behaviour as well as for the presentation of recommendations (in specific situations as well as in an ambient way). We analyze the technical capabilities of mobile phones that can support the creation of persuasive technologies. The focus of the work is to explore the domain of ambient displays and persuasive technology with regard to communication. We present designs of how the screen saver on a phone can raise users' awareness of their personal communication behaviour. Additionally, we show how this concept is applicable to further application areas such as increasing the awareness of the user on how his time is spent or creating stronger bonds in peer groups.
\end{abstract}

\section{Categories and Subject Descriptors}

H5.2. User Interfaces, D2.2 Design Tools and Techniques, H5.m. Information interfaces and presentation (e.g., HCI): Miscellaneous.

\section{General Terms}

Design, Experimentation, Human Factors

\section{Keywords}

Ambient displays, mobile devices, information visualization.

\section{INTRODUCTION}

In many parts of Asia, Europe, and America mobile phones are used commonly in daily life to communicate, coordinate and to access information. The majority of people in these areas carry a phone with them at most of the time. If they do not have it on the body then it is within close reach [4]. Due to the usage in various different kinds of situations, mobile phones function as probes of

Permission to make digital or hard copies of all or part of this work for personal or classroom use is granted without fee provided that copies are not made or distributed for profit or commercial advantage and that copies bear this notice and the full citation on the first page. To copy otherwise, or republish, to post on servers or to redistribute to lists, requires prior specific permission and/or a fee.

Mobility'06, October 25-27, 2006, Bangkok, Thailand.

Copyright 2004 ACM 1-58113-000-0/00/0004 ...\$5.00. the users' everyday life environments. Mobile phones contain communication logs for messages and calls as well as user-created content, such as calendar notes and photographs. These features make a mobile phone a versatile storage of personal user data.

Mobile phone usage has created cultural trends of its own, especially among teenagers [7]. Numerous ways for personalizing the phone are a result of this phenomenon. Choice of the model, changeable covers, ring tones and wallpaper selections are commonly used to show personal preferences or as part of the image the user wants to reflect.

Interaction with the phone and through the phone reveals and reflects on the user's behaviour. Although mobile phones are commonly used in public, they are perceived as very personal items [3]. The users do not tend to reveal to others the personal information contained in the phone, and privacy of this data is highly valued. With regard to younger users (teenagers), initial interviews reveal that communication meta-information is regarded as very private information. Sharing of this information requires trust. Typical examples of such information are:

- Number of SMS received from a certain person

- Persons who called recently

- Persons to whom outgoing calls were made recently

Similarly, information on the whereabouts of the user can be detected with the phone, at least on a coarse scale. As the phone is nearly always with a user, the location of the phone reflects the places where a user has been. Collecting information on the location of users over time and presenting it to increase awareness of their activity appeared as a further interesting issue from our initial investigations. An assumption is that providing users with information about where they were for what period of time can help them to reflect on their behaviour.

In this paper, we present a design study that investigates how mobile phones can be used as sensors and unobtrusive presentation devices to increase users' awareness with regard to communication and location. In Section 2, we show how mobile phones can be used as sensors for communication and location. Then we investigate options for presenting information on the phone, taking into account concepts of ambient visualisation and information art. We have conducted a design experiment with 13 teams looking at options for visualisations to increase communication awareness, which is described in Section 4 . We then present further examples of how this concept can be used in applications. In Section 6, we report results from a series of user 
interviews that have been conducted in Germany and Finland. We then conclude the paper with a discussion of the results.

\section{MOBILE PHONES AS SENSORS}

Current mobile phones contain a lot of information that can be used to reflect on the user's activities, communication and location behaviour. Considering the use of mobile devices and research that assessed the inclusion of sensors into the phone [11], the following parameters can be used as a source of information for applications:

- Activity with the device (e.g. calling, messages),

- User location (e.g. based on cellular-id or GPS information)

- Co-location (e.g. proximity to other devices)

- $\quad$ Physical activity (e.g. sitting, running).

In this paper, we concentrate on information that is available in existing phones on the mass market. The focus of the design cases presented later in this paper is on the communication metainformation (e.g. number of calls made and received, number of messages sent and received, communication with a certain person) and on cell-based location information.

\subsection{Communication Behaviour}

Meta-data on communication is typically available on the phone in a list showing recent calls, missed calls and calls received. Similarly,, the information about sending and receiving messages can be extracted from the sent folder and the inbox of SMS and MMS. Additionally, many service providers make the information about outgoing and incoming calls available in the form of itemized bills which can be downloaded from the user's account with the provider. Such itemized bills usually include further information, such as duration and cost of the call. For prototyping, meta-data on communication can be traced either from the phone or by accessing the online itemized bill. For a deployment of such a concept in the large we would expect that one model is that the service provider offers such a screen saver as an additional service (and basically selling the itemized bill in a different visual appearance).

It can be assumed that the communication patterns represent information which the user him/herself is interested in both for affective and practical reasons, but which is also considered to be private as outlined before. Communication logs reveal information not only about who is contacted regularly, but also how communication patterns (frequency, duration, content length) change over time for a certain person. Thus, communication logs offer a potential information source for information art presented in an ambient manner. To explore the possibilities, we constructed a design exercise with about 50 students in an HCI class which is described in a later section.

For the interviews that were carried out, we have evidence that information on one's communication behaviour is of interest, but that people hardly make the effort to trace such information for the available sources. People consider it interesting to know how much time they spent talking to their partner and how this information changed over recent weeks. However, at present this information is not easily accessible to them. In our interviews, it appears that there is a general agreement that increasing the awareness of communication patterns is of interest and can help the user to reflect on his behaviour.

Visualisation of data related to communication can help to increase the user's awareness and hence make it a persuasive technology being an incentive for certain behaviour [2]. If one thinks close contact to parents is important, ambient visualisation can become a central means providing this awareness.

\subsection{User Location and Activity}

The phone can provide information about the user's location in the physical world. Depending on the technology used, the location information is available with a different level of detail. All mobile phones, when operational for communication, know which base station they are connected to. This can provide a location cue. In most cases, the cell ID information can provide meaningful information despite its coarse resolution - for example, most often the cell ID for the home differs from the cell ID of the workplace. Moving between places can be detected by monitoring changes in the cell ID and signal strength. Storing the history of visited cell IDs and the frequency and duration of visits provides a basis for reasoning about the user's behaviour.

To implement this, access to information on cell ID and signal strength is required. Not all phones provide this information to the programmer today. For prototyping, we used Phyton for Nokia Series 60 which provides easy access to the cell ID and signal strength. Another option is to use JAVA J2ME, which specifies a location API. However, this is still not supported on many phones. Using more advanced reasoning based on a set of reachable cell IDs in the vicinity can provide a more precise location, as demonstrated in [6]. Some phone models also include GPS which can yield higher-resolution location data in outdoor settings.

Location is the key information that can help users to remember what they were doing. Similarly, location patterns and changes in these patterns provide information on the user's activities. An initial idea, which is further explored in the design studies, is to look at the time spent in certain locations. We assume that even an abstract presentation of this information will increase users' awareness of their whereabouts; one example is to provide information on the percentage of time spent at home, in the office, and travelling. Looking at statistical patterns in locations visited reveals answers to questions such as:

- Have I been to a new place during this week?

- Have I spent more time with my family this week than the weeks before?

Co-location information describes encounters with mobile units (other users) and stationary objects (equipment in the office). This can be detected by using short-range communication technologies, such as Bluetooth or wireless LAN. Encounters allow deducing fine-grained information on locations visited and persons met. Colocation information can be used for tracking social patterns and places where people meet.

Physical activity can be detected with accelerometers integrated into the device. Several phone models also have the ability to measure environment temperature. Using the built-in camera and microphone, further information about the environment can be detected, e.g. whether is it noisy.

\section{PRESENTATION ON THE PHONE SCREEN}

Most mobile phones have one or two screens that allow the presentation of a small amount of information. When the phone is not operated (e.g. no phone calls are made, no messages sent or received) the screens are not required for any use. In idle mode, the screens are off or show the time and date, small animation, a picture or any other screen saver. Sometimes, small overlaid icons are used to indicate that there were missed calls or messages that 

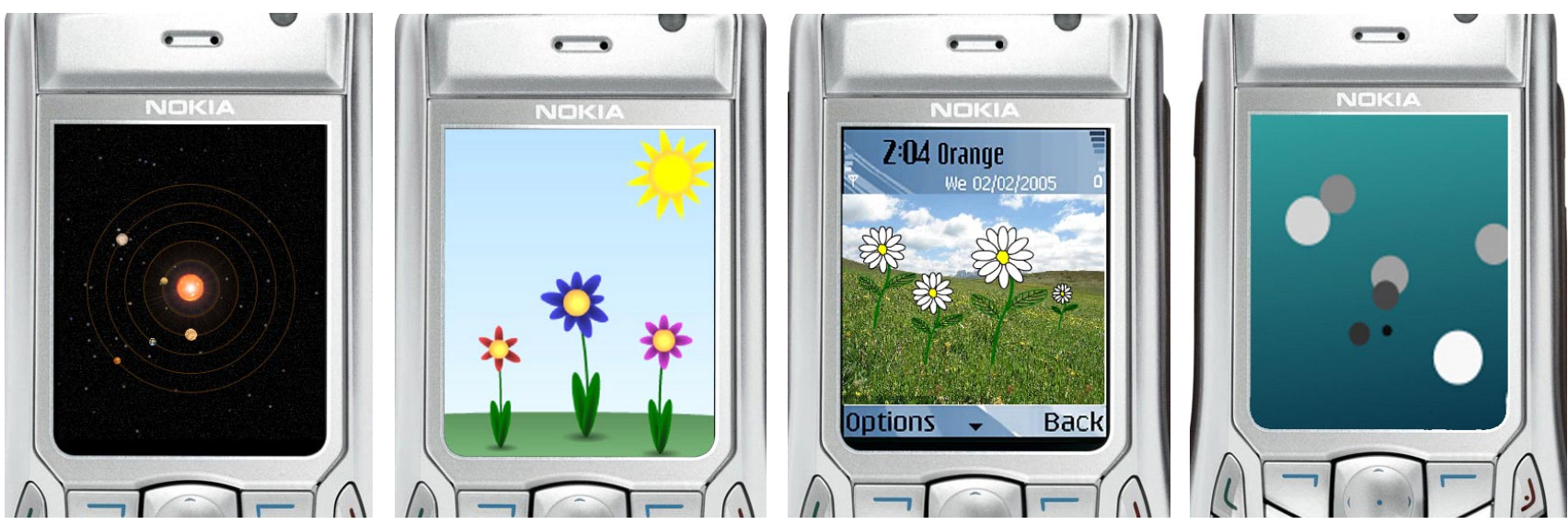

Figure 1. Four designs of screen savers on mobile phones. Each can be used as an abstract way of showing information about frequency and length of calls to certain contacts. A contact is mapped to a planet, flower, or disc, respectively.

Users can then get an idea of frequency and length of phone calls at a glance by looking at size and speed of the objects.

have not yet been read. In the case of an incoming message or call, the screen is used by the application, usually to show the caller's ID, contact information, or picture.

As phones are used in social settings, the information presented on the screen in the idle state is not fully private. One example is that people put their phone on the table while having a meeting or lunch. In this case, others can see the content of the phone's display. Therefore it is important to preserve the user's need for privacy when designing information that is displayed on the phone screen - it cannot be assumed that the screen is only seen by the user.

In our project, we explored the idea of information art and using the mobile phone as ambient display to visualise communication and location behaviour, as described in the next section. Another option for presenting information on the phone is to explicitly trigger and present information in a certain situation and alerting the user to take notice. This phenomenon is briefly discussed in the second part but not further investigated.

\subsection{Implicit - Creating Awareness}

The basic idea for implicit information presentation is to use a screen saver to show information about behaviour or other information that the user would like to be aware of. As this information is very personal, it is not appropriate to visualise it in a way that is obvious to everybody. This is the initial rationale to further investigate the concept of ambient information visualisation [5][9][10]. Ambient displays are displays which present information on the periphery of the user's attention in an aesthetically pleasing manner, without distracting or straining the user [8]. Even though smart phones offer a variety of additional applications, reaching from address books to video playback, their potential as a platform for ambient information display has not yet been utilized. Nonetheless, they represent a potential platform for such applications by offering various kinds of input data, sufficient computing power and display capabilities. The existing personalization culture also suggests that users are willing to customize their devices and are open to receive personalized information.

Previous research which introduced information art has concentrated on public spaces and large physical elements [7]. An example of personal information art is an ambient display, which shows the status of some user-defined information, e.g. traffic or temperature figures as arbitrary elements on a wall display [12] Information visualisation for large audiences has for example been demonstrated with the IN-Visible system, where an ambient representation of subway information is displayed in public urban spaces [1]. Personal mobile devices have so far not been considered as a platform for information art.

The focus of our research is on how to use the phone screen as an unobtrusive ambient display that fits into the usage patterns and that makes the phone more interesting and pleasant to look at.

\subsection{Explicit Reminders That Suggest Actions}

Apart from acting as ambient displays, phones are also an attractive platform for explicit reminders. As the phone is very often accompanying the user and used to acquire information about situations based on location, co-location and activity, it becomes feasible to trigger context-dependent reminders.

The following two scenarios highlight this potential. A user has decided to improve his fitness. The phone can then be used as a reminder of this resolution and provide information in context that helps to achieve this goal. When the user approaches an elevator, it could suggest taking the stairs. Similarly, when a user decides to communicate more with her parents she could be reminded to call them while waiting at the bus stop.

In general, the information presentation for persuasive applications is most efficient in the situation where it is most applicable and where users have a chance to act and change behaviour. These types of persuasive applications are intriguing but place many questions well beyond the technology. We will explore this in further work.

In the next section we concentrate on ambient visualisation of the user's communication behaviour on the phone screen.

\section{DESIGN EXPLORATION OF AMBIENT DISPLAYS USING MOBILE PHONES}

In a large design exercise, we investigated the options for visualisation of the communication behaviour on the phone. Using communication $\operatorname{logs}$ as the basic data, we started to explore potential designs for ambient information displays. We assumed in this design phase that the information that is typically present in an itemized bill (time and cost of all calls that were made, all messages sent and associated cost) as well as information about incoming calls (as in the call lists of the phone) is available. 
After a number of initial informal interviews with potential users and developers of mobile phone applications, we decided to prototype the concept. To investigate the potential design space, we gave 13 teams of 3 to 5 people each the task of designing a screen saver and a setup screen for the given task. The participants were mainly students of computer science and media informatics at the University of Munich in Germany. In a time frame of about 3 weeks, they were expected to create sketches and a functional prototype (on a PC) of their concept. Requirements were that the visualisation can be customized and has the properties of an ambient display.

Personal feelings, emotional situations (such as happiness or problems) and ongoing activities reflect on our communication behaviour. The task was that the application should enhance the user's ability to follow the overall communication activities and to keep track of the frequency and intensity of the communication with different people. Additionally, changes in the behaviour over a period of time could be included into the designs.

The teams were free to add further elements as parts of the visual design. The temporal resolution (e.g. communication happening daily or weekly) could be incorporated in the visualisation by means of colours, shapes, or other details. The deviations in the visualisation show the changes over time, e.g. when the current visualisation is compared to the one from last week or last month.

The following design cases on ambient visualisation of communication behaviour illustrate the results. We selected some example designs that provide peripheral awareness for personal communication patterns. Those examples are suited to show the potential design space and the options available for the ambient visualisation of personal communications behaviour on a mobile phone. Screenshots of the introduced designs are presented in Figures 1 and 2. The animated designs of the prototypes are available on our website at http://www.hcilab.org/ambientphone/ .

\subsection{Design Case A: Solar system}

This prototype uses the solar system to visualise the information, see the leftmost picture in Figure 1. The planets are all constantly moving around the sun (the centre). In the initial prototype, each planet represents one communication partner. The size and velocity of the planet reflect the communication events. The bigger the planets, the more messages were exchanged, and the faster the planet moves, the more call conversations have taken place with the person. In the setup screen of this prototype, contacts can be assigned to planets. For example, one can assign one's best friend to the Earth and one's grandmother to Mars. The communication behaviour with these people is then reflected by the components in the visualisation.

This visualisation offers further parameters that could be used to map information, e.g. activity of background stars, rotation of planets or appearance of comets. During the discussion of the result, one idea was to add a comet automatically to the visualisation if there is a lot of communication with a new person. The user would then be able to look up the respective communication partner in the setup screen and potentially map this new contact to a planet.

\subsection{Design Case B: Aquarium}

The aquarium prototype reuses a common screen saver scheme. Each chosen communication partner is represented by a fish; see Figure 2 on the left hand side. The fish are animated. In this prototype, the size of each fish reflects the money the user spent on communication with this person; the bigger the fish the more money was spent. The speed of the fish is related to the time since the last call. If the fish is very slow, there was no communication for a long time. The direction of the fish visualises the direction of the initiation of calls. If the fish swims from left to right, you call more often than you are called by this person.

Options available to convey further parameters are bubbles from the mouth of a fish, the animation of the mouth of the fish, and plants in the water.

\subsection{More Design Examples}

In the picture on the right in Figure 1, an abstract representation is used. Each circle (distinguished by colour) is a person and the size and speed of the circle represents communication metainformation for this person. Likewise, each circle can be used to represent one call. The colour links it to a person and the size shows the duration of the call.

The flowers of the two designs in the centre of Figure 1 are similar in concept to the fish in the aquarium. In the basic version, only the size of the flower is used to represent the communication activity with the respective person over the last weeks. In the setup screen, a contact can be assigned to each flower. The scene includes a sun that is used to communicate further information. A setting sun can be mapped to specific communication events; similarly the intensity of the sun is a further parameter that can be mapped to information (e.g. overall communication behaviour or battery status).
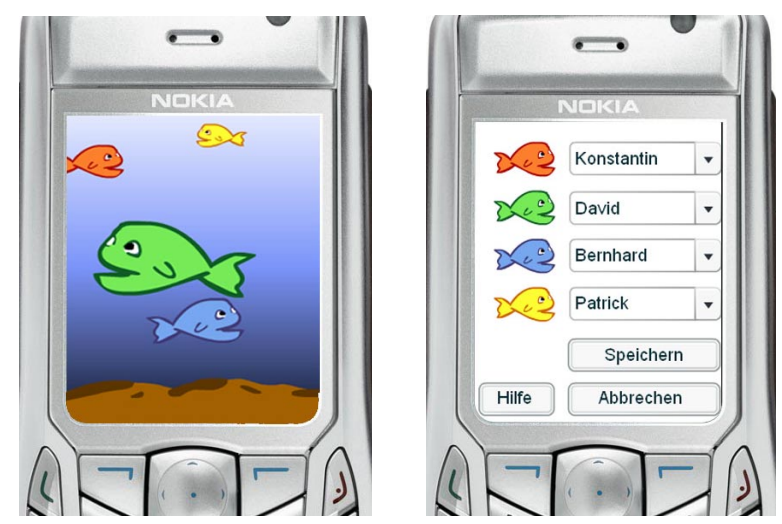

Figure 2. A screen saver where coloured fish are mapped to contacts and the corresponding configuration dialog.

Parameters like speed, size and direction of the fish can be used to show communication patterns.

Creating a large number of prototypes by a diverse group of people proved to be a very effective way of generating ideas and assessing the design space for this type of application. The designs shown are prototypes implemented in Macromedia Flash MX and Java running on a PC. An implementation of prototypes for use on phones is currently under development. Additional studies on the topic have been designed and will be carried out in the near future. Our interest is in particular on how users want to customize their personal system (e.g. what options for association between contacts and visualisation are preferred). The other area under investigation is concerned with the privacy requirements for the visualisation. In particular, we will look at what level of abstraction in the ambient visualisation is appropriate to allow the users of the phone to be aware of the parameters without making it too easy to guess for others. 

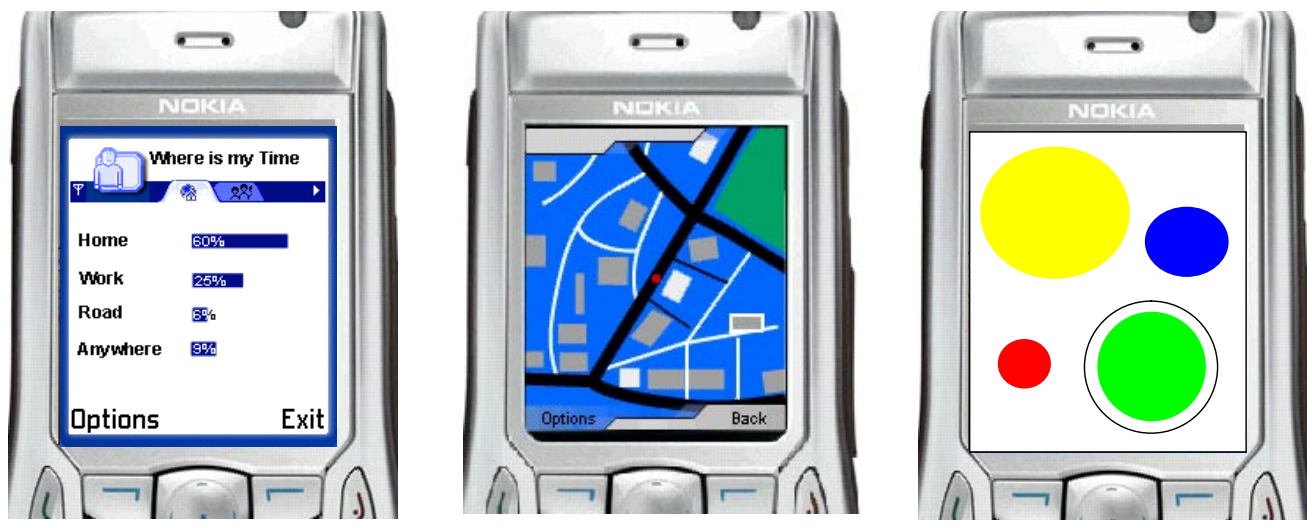

Figure 3. Three designs that show how much time people spent at specific locations. The leftmost application shows a list with percentages that can be explicitly opened through the phone's menu. The other two designs are screen savers that highlight important locations on a map or visualise long stays with larger coloured circles.

\section{VISUALISING LOCATION AND ACTIVITY}

Beyond communication, we have investigated further application areas. Recalling where one has been seems for most people a task that is rather easy. However, estimating the amount of time spent in certain locations is more difficult. Even though they appear simple, the following questions are difficult to answer for many people:

- What percentage of your time did you spend at university?

- How much time did you spend at home last week?

Answers to these questions have the potential to make us more aware of our activities. With the following prototype designs, we try to increase the users' awareness of their location behaviour.

The basic approach is to use the cell ID as the basic location information and to map the main locations to meaningful places for the user, such as home or work. This mapping may be explicit (e.g. by giving names) or implicit by just highlighting the current cluster. Rapid changes in signal strength and cell IDs are used as an indicator for travelling. By keeping track of locations (cell IDs) seen, we are able to accumulate the time spent in certain locations.

The three screens in Figure 3 show the major design options we are interested in. In the first screen, an explicit non-ambient visualisation can be seen, showing the percentage of time spent at home, work, travelling, and elsewhere. The second screen is an ambient visualisation of an animated map where the user's main locations are visualised, and the third example is an abstract visualisation of the four categories. The size of the circles relates to the percentage of time spent in that location, and the current location is highlighted. No explicit naming is required here as the users can tell which location is which by observing the highlighted location. The different options were discussed with users in interviews; see Section 6 for the results.

\section{EVALUATION}

To evaluate the overall concept and the specific designs, we conducted 20 interviews in Finland and Germany. In the following, we outline our methodology and discuss our results.

\subsection{Methodology}

Our primary goal was to evaluate whether people understand and like the proposed concept. The benefit of being more aware of one's communication behaviour or one's whereabouts is personal and subjective. We do not aim at making people more efficient or productive with the system introduced, but rather to increase their awareness and their overall satisfaction with regard to communication.

Our method of choice was to conduct structured interviews based on a set of questions. The questionnaire and the raw results can be found on the project page page http://www.hcilab.org/ambientphone/ .

Each interview was structured in three phases. First, we asked general questions about mobile phone usage, personalisation and communication behaviour. Then we showed and explained the prototype designs and asked specific questions relating to these designs. In the final part, we had a more open discussion with the participants on the concept of using mobile phone screens as ambient interfaces. The final part was explorative and designed to create new ideas.

\subsection{User Interviews and Results}

Based on the questionnaire, we conducted 20 interviews. Each interview took between 15 and 40 minutes. The selected participants were mainly people who have owned and used a phone for several years.

Of the 20 people who took part in the interviews, 12 were male and 8 female. The majority of them were between 20 and 30 years old (14), 5 between 30 and 40, and one older than 40 years. We asked 9 students, coming from the field of informatics, media informatics and social sciences. Others had jobs in the industry as land surveyor, project manager as well as software engineering, electronics, user interface design and other IT sectors.

The interviews took place at various places, most of them in meeting rooms or offices at a university or company. We met 7 people at their homes and carried out one interview on the phone, and two via emails assisted with phone calls. People from three different countries took part: Austria (2), Germany (10), and Finland (8). All of them have been using a mobile phone for at least 2 years.

The first part of the interview was concerned with general questions on mobile phone usage and awareness of users with respect to communication and location behaviour. After collecting information about whether and how people customize their 
phones, we presented them our applications. The second part then concentrated on getting feedback on these designs.

Nearly all interviewees (17 of 20) use their mobile phone regularly or at least several times per week to call or receive messages. The same applies to the number of people who use the short message service (SMS) to send and receive messages.

We were particularly interested in seeing whether people are customizing their mobile phones. We found that all participants store most or all of the phone numbers (several times together with accompanying information like address and birthday). More than half of the interviewees also saved some or many pictures on their phones, like pictures embedded in their contact lists or the photos taken with the camera of the phone.

Although only 3 mobile phone users had a non-standard ring tone, only 4 had kept the factory setting or were happy with it. The others picked another one from the menu, downloaded or bought one (2), or they created one by themselves (2). Only 3 people did not change the setting of the colour scheme and background (if it was possible to change them at all). The rest of them either picked another available background from the menu or had a photograph as a background picture.

From our perspective, it was very interesting that, in contrast to the willingness to customize several parts of the mobile phone, there were only 3 people who changed their screen saver to something other than displaying the time or nothing at all. Just 10 per cent used a self created logo or picture as screen saver. Of course, this finding is restricted by the fact that various phone models do not offer the possibility to personalize the screen saver. Apart from the time and sometimes icons for missed calls and received SMS, no screen saver showed additional information.

Very important questions for our application were whether or not people knew about their communication behaviour in a longer period of time and whether this is of interest to them. Less than half of the interviewees ( 8 of 20) stated that they were confident about with whom, when, and how long they had talked on the phone during the last months. Another 7 people thought they might have at least a vague idea of their communication patterns. The average number of people with whom users talk longer and regularly is quite small (4.6 with a small standard deviation of 2.2) and this helps to get a rough idea. In addition, people seemed rather interested in having information on with whom and how long they talk on the phone, and 7 out of the 12 people who paid their bills themselves read their itemized phone bills in detail and not only to check if the total amount is correct.

Their opinions on whether people would be influenced by such data on communication patterns were highly diverse. Still, 9 users stated that they think that at least their awareness of their relationships might increase. In several of the interviews, participants even said they could imagine that they would call someone more often if they saw they spent very little time talking to this person, or reduce the number of calls in other cases. Only one said that she would see no difference at all. A clearer result was obtained when talking about privacy: Over 90 percent of the users found that information about their communication behaviour is private and should not be available at all to others; 13 of 20 even strongly emphasized this consideration.

After showing and explaining two example designs (see the design on the left in Figure 2 and the one in Figure 3), we were assured that only 2 people had slight doubts regarding privacy issues. These could be eased by using a password protection to see and alter the mappings when giving the phone to someone else.

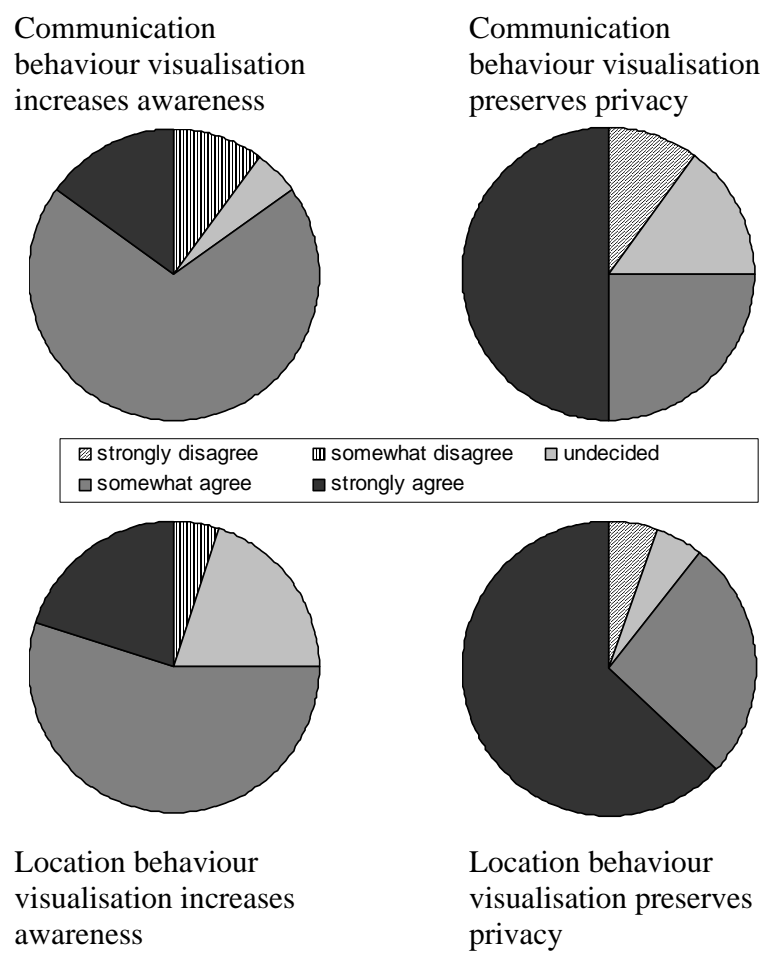

Figure 4. Users stated that the screen saver designs clearly increased their awareness of communication and location behaviour while at the same time preserving their privacy.

As illustrated Figure 4, the vast majority of participants $(85 \%)$ rated the applications as very useful to increase the awareness of their communication behaviour. The users also confirmed our assumptions during the design by giving an average of about 6 contacts to be visualised (neglecting one person who suggested $50)$. We were pleased to see that only one interviewee clearly did not want to have such a screen saver but 15 users directly expressed that they would like to get one. In 16 cases we were told that users would love to try or be interested in further options for customisations in general if it was of some use to them.

Along the same lines, we queried people with respect to location behaviour. We asked the same questions as before regarding communication and got comparable answers. A majority (14) was confident that they knew the distribution of where they spend much of their time, but nevertheless found it interesting to be aware of where they spend time. There was an average of 4 to 5 locations that were of particular interest for a visualisation like the ones shown in Figure 3. After showing these three designs to the participants of the interviews, we asked for opinions on them. It became clear that such a visualisation increases the awareness of where people spend their time (15 positive statements, see also Figure 4) and that many users would like to have such a screen saver. The concept of the application shown as the leftmost 
picture in Figure 3 that must be explicitly opened to see the resulting list was preferred to the screen saver models shown in the other two pictures by half of the interviewees. Their arguments were based on the fact that this kind of information might not be needed or of interest so often, especially not before or after using the phone, and also because of the privacy issue with a presentation employing a map. In addition, the detailed display fascinated some of the users. However, the other half preferred the screen savers, especially the abstract version shown on the right in Figure 3. The version in the centre, showing fragments of maps highlighting locations where the user spent much time seems too complicated as an abstract representation and not as secure with regard to privacy. The one with coloured circles provides a quick and colourful overview and can be used as a screen saver since it preserves the privacy (so say 17 of 20 persons). Details like percentages could be easily added to implementation if required.

In total, we had a very positive experience with all of the participants of the interviews. Everyone very quickly seemed to grasp the idea of using the mobile phone screen saver as ambient display and we received a lot of positive remarks. We saw that data about communication and location is judged to be very private information but also quite interesting to know about. The important factor for such designs is to keep the presentations abstract enough to ensure that others cannot easily find out private information. A design like the map shown at the left hand side of Figure 3 would not have triggered such convincing answers as indicated in the two pie charts on the right of Figure 4. Users also saw much potential in using this idea for other application areas like visualising special events or health data. Some interviewees were surprised that they do not know about any screen savers that convey additional information in an ambient and unobtrusive way.

\section{CONCLUSIONS}

Mobile phones are a widely deployed platform and for many people a central instrument for communication. We have experienced that using the phone screen for conveying information of interest to the user in an ambient way is feasible and interesting. Our initial designs show that especially the phone as a mobile companion can become a meaningful ambient display and potentially a platform for further persuasive technologies.

The usage model of mobile phones makes them suitable for use as ambient displays. The personalisation culture (currently mainly background images and ring tones) invites further investigation of means for personalisation. The design examples shown in this paper lay out a design space for novel services where information of interest to the users is visualised.

Our interview-based evaluation supports the assumption that personal information presented in an ambient way offers a potential application area for mobile technologies, as the concept was well understood and the idea was found appealing. Although the evaluation consisted of only 20 interviews, we believe it gives good indications on how people perceive the idea of using mobile phone displays for ambient visualizations of personal data. As privacy was considered very important, special attention should be devoted to the design of the visualizations. We also learned that using some sort of known scenery as the background or fundament of a design is slightly preferable to entirely abstract designs. The students who made the designs of the screen savers often remarked that they found it easy to parameterise and use existing objects and users tended to like nice settings where dynamic objects are smoothly embedded, as they could more easily imagine having a nice picture as background image or screen saver.

\section{REFERENCES}

[1] Chatzitsakyris, P., Decla-Soares, G., and Zulas, A. INVisible: Perceiving Invisible Urban Information through Ambient Media. In CHI 2004 Late Breaking Results Paper, 1348-1350.

[2] Fogg, B.J. Persuasive Technologies. Communications of the ACM, 42(5): 27-29, 1999

[3] Häkkilä, J., and Chatfield, C. (2005). 'It's Like If You Opened Someone Else's Letter' - User Perceived Privacy and Social Practices with SMS Communication. In Proc. of MobileHCI 2005.

[4] Ichikawa, F., Chipchase, J., Grignani, R. Where's the Phone? A Study of Mobile Phone Location in Public Spaces. In Proc. IEE Mobility Conference 2005 in Guangzhou, China, $797-804$.

[5] Ishii, H., Ullmer, B.: Tangible bits: Towards seamless interfaces between people, bits and atoms. In CHI'97. (1997) 234-241

[6] LaMarca, A., Chawathe, Y., Consolvo, S., Hightower, J., Smith, I., Scott, J., Sohn, T., Howard, J., Hughes, J., Potter, F., Tabert, J., Powledge, P., Borriello, G., Schilit, B.. Place Lab: Device Positioning Using Radio Beacons in the Wild. In Proc. Pervasive 2005: 116-133

[7] Ling, R. The Mobile Connection. The Cell Phone's Impact on Society. Elsevier Inc., 2004.

[8] Mankoff, J., Dey, A. K., Hsieh, G., Kientz, J., Lederer, S., and Ames, M. Heuristic Evaluation of Ambient Displays. CHI Letters, Vol. 5, Iss. 1, 169-176, 2003.

[9] Pedersen, E. R., Sokoler, T. AROMA: Abstract representation of presence supporting mutual awareness. In Proc. CHI'97, ACM Press (1997), 51-58.

[10] Redström, J., Skog, T., and Hallnäs, L. Informative Art: Using Amplified Artworks as Information Displays. In Proc. of DARE 2000, ACM Press, 103-114.

[11] Schmidt, A., Aidoo, K.A., Takaluoma, A., Tuomela, U., Laerhoven, K.van, and Velde, W. van de. Advanced Interaction in Context. In Proc. Of the First International Symposium on Handheld and Ubiquitous Computing (HUC99), Springer LNCS, (1999), 89-101.

[12] Stasko, J., Miller, T., Plaue, C., Pousman, Z., and Ullah, O. Personalized Peripheral Information Awareness through Information Art. In Proc. of Ubicomp 2004, 18-25 